\title{
Calculations for the Rheology of Foam Fracturing Fluid
}

\author{
Xiao Sun ${ }^{1, a}$, Lin Huashen ${ }^{2, b}$ \\ ${ }^{1}$ Research Institute of Yanchang Petroleum(Group) CO. LTD, Xi'an 710052 \\ ${ }^{2}$ School of Energy and Power Engineering, Xi'an Jiaotong University, Xi'an 710049, China \\ aycyjysx@163.com, bguoren1984@163.com
}

Keywords: Rheology; Foam fracturing fluid; Eulerian-Eulerian two-phase fluid model.

Abstract. Eulerian-Eulerian two-phase flow model was used to calculat the rheological properties of foam fracturing fluid. In the model, the foam fluid is treated as pseudoplastic fluid. And the calculation results of the model show that the non-Newtonian behavior of foam fracturing fluid is determined by the bubble phase, and there is almost no slip between liquid phase and bubble phase. The turbulent kinetic energy of each phase increases with increasing volume fraction of gas phase and has the largest value at the wall adjacent region. However, this two-phase fluid model is not suitable for foam fracturing fluid with gas volume fraction higher than $65 \%$.

\section{Introduction}

Foam fluid is widely used in industry [1], such as well stimulation and cleanout, liquid-mobility control, acidization, water shutoff and gas blocking in water-coning prevention, gas storage, and so forth. So up to now, much attention was paid to it. But most foregoing researches were programmed by experiment and numerical simulation studies were less, because foam fluid is a kind of rather complicate discrete system with gas phase dispersed in liquid phase.

For general foam fluid, simulation studies on its rheology have been taken by some researchers. Durian [2] proposed a simple model by focusing on entire bubbles rather than films or vertices for the deformation and flow of foam. Yuan and Edwards [3] used the vertex model to research "dry foam" whose foam quality was close to $100 \%$ by. Simon et al. [4] and Kern et al.[5]did research on the same thing using viscous froth model. Sun and Hutzler [6] introduced a discrete two-dimensional hybrid lattice gas models to research "wet foam". Besides, to illustrate the effects of surfactant on the rheological properties of emulsion and on the dynamics stability of evolving microstructure, Pozrikidis [7] simulated the evolution of surfactant concentration by the method of interfacial dynamics combined with an implicit finite-volume method.

\section{Eulerian-Eulerian Two Phase Fluid Model}

When foam quality is over 0.5 , the viscosity of foam fracturing fluid will increase rapidly. If one uses drift flux model or two-fluid model to simulate the rheology of this two-phase fluid, the increase of effective viscosity of foam fracturing fluid is caused by the interaction of every term of nonlinear momentum. But the slip velocity of the two-phase is rather small in the foam fracturing fluid. Hence, it's impossible that the nonlinear energy dissipation between liquid phase and gas phase leads to the steep rise of effective viscosity. In fact, the viscosity of foam fracturing is mainly related to the inter-collision and inter-friction of bubbles. Therefore, here we adopt Eulerian-Eulerian two phase fluid model, which treats the bubble as pseudoplastic fluid to simulate the rheology of the foam fracturing fluid.

Supposing that there is no mass transfer between liquid and granular phases, the mass conservation equations of the two phases are as follows:

$$
\frac{\partial}{\partial t}\left(\alpha_{l} \rho_{l}\right)+\nabla \cdot\left(\alpha_{l} \rho_{l} u_{l}\right)=0
$$




$$
\frac{\partial}{\partial t}\left(\alpha_{b} \rho_{b}\right)+\nabla \cdot\left(\alpha_{b} \rho_{b} u_{b}\right)=0
$$

As for fine bubbles, the buoyancy force can be ignored. Therefore, the momentum equations become:

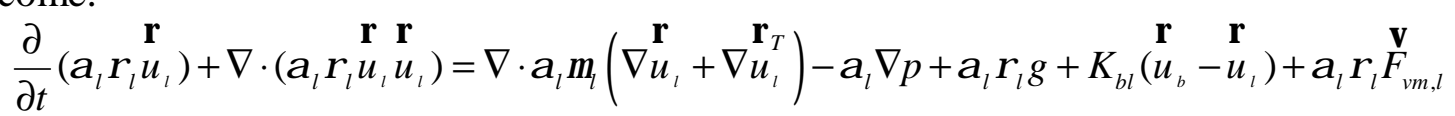

(3)

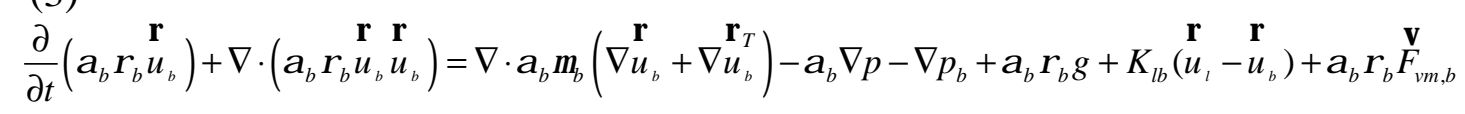

\section{Simulation results}

Effective viscosity

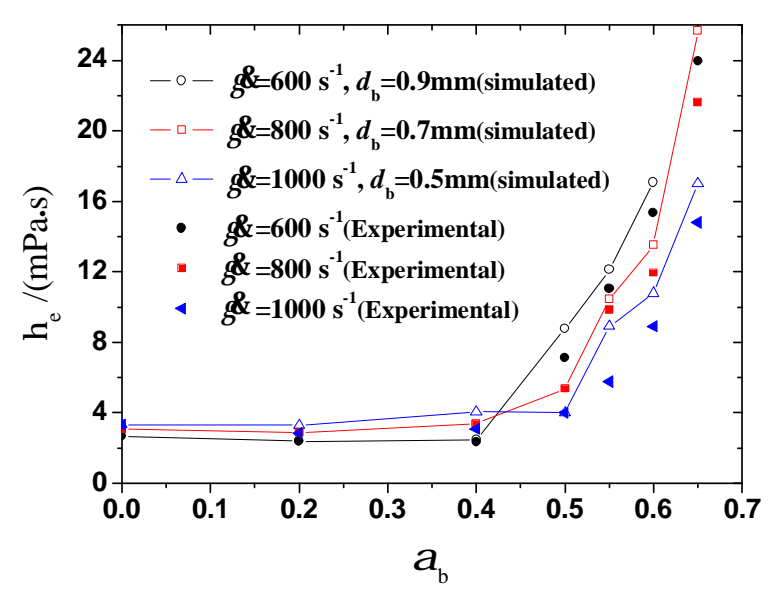

Fig.1. Effective viscosity changing with foam quality and shear rate

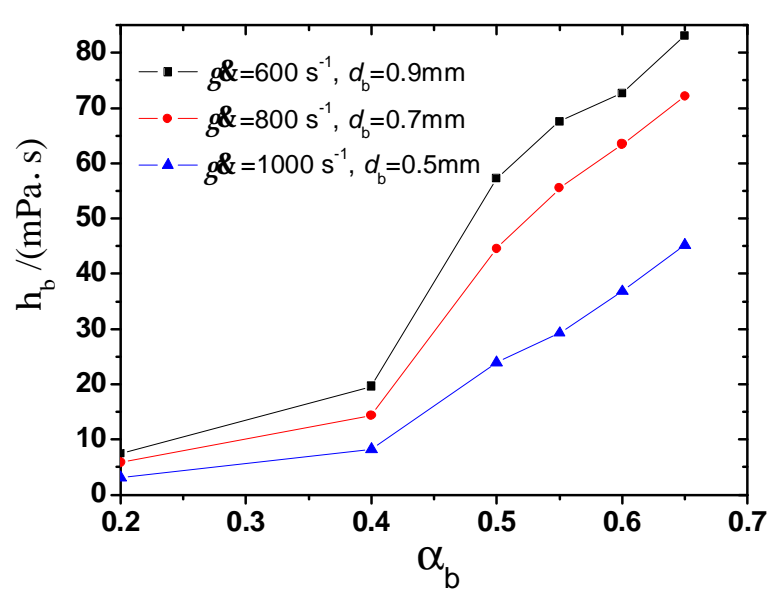

Fig.2. Viscosity of granular phase changing with foam quality and shear rate

Fig.1 shows the effective viscosity changing with foam quality and shear rate, in which the bubble diameters in different shear rates are obtained from Harris. It's found that the simulating result is a little bigger than that of experiment. It probably because the simulation considers only bubble's movement, collision and friction, but not distortion and breaking up of bubble which take place in real fracturing fluid.

Viscosity and ponderance

Fig. 2 shows the variation of granular viscosity $\eta_{b}$ changing with foam quality and shear rate. The viscosity and the non-Newtonian behavior of foam fracturing fluid is determined by the bubble phase.

From Eq. (10), the viscosity of granular phase is caused by bubble's movement, collision and friction. When foam quality $\alpha_{b}$ is small, collision is the main influencing factor. But $\eta_{b}$ isn't high because the bubble number is small and the bubbles are far apart from each other at that time. With the rise of foam quality bubbles collide with each other repeatedly, which results in energy dissipation and $\eta_{b, c o l}$ increase. Besides, the higher the foam quality, the more bubbles, which leads to tight bubbles and greater friction energy dissipation. When foam quality is over $60 \%$, energy dissipation caused by friction is at the dominant status (Fig.3). 


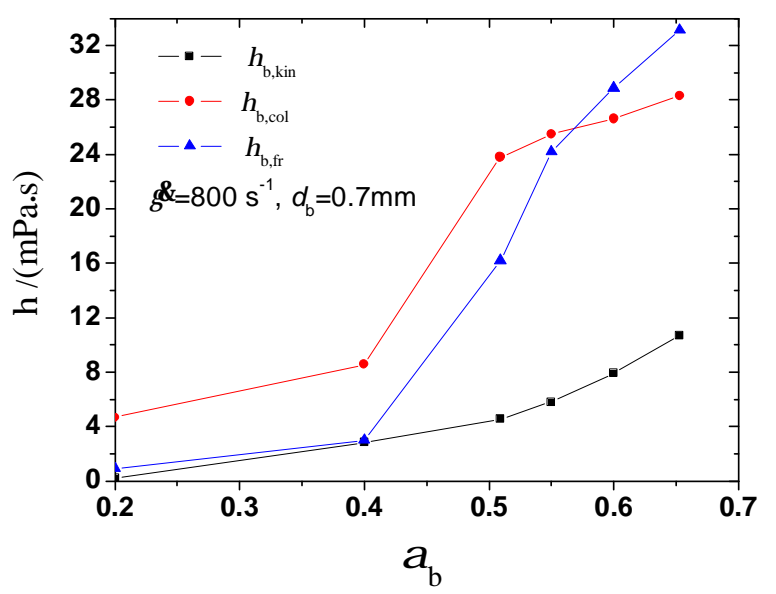

Fig.3. Viscosity of granular phase caused by movement, collision and friction

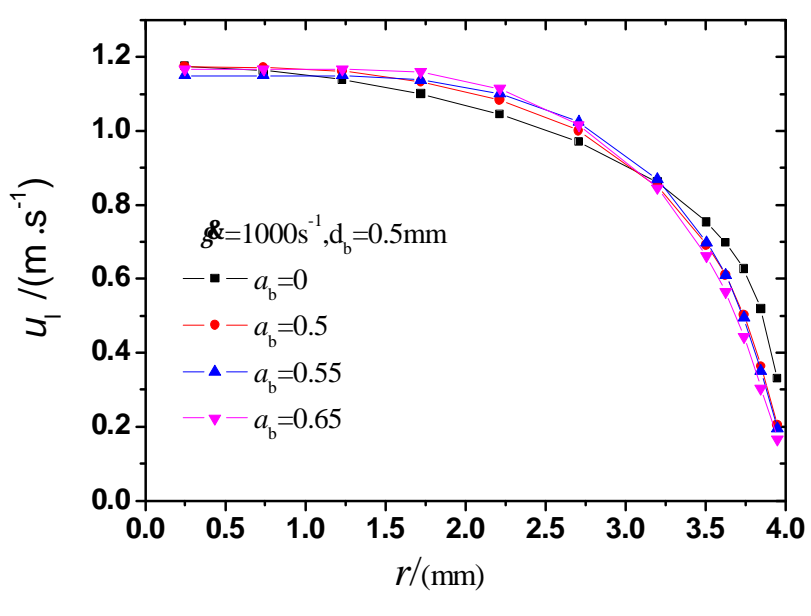

Fig.4. Velocity profile of liquid phase

\section{Velocity profile}

The velocity slip between liquid and granular phases is small in stable foam. The experiment result of Амиян shows that when the bubble diameter equal to $1.2 \mathrm{~mm}$, the slip velocity of a bubble in water is $30 \mathrm{~cm} / \mathrm{h}$, and that in aqueous foam with $1 \%$ surfactant is $8 \mathrm{~cm} / \mathrm{h}$. Figs. 4 and 5 present the velocity profile of liquid and granular phases respectively. It can be seen that the velocity slip between the two phases is rather small, which is similar to the results of Амиян.

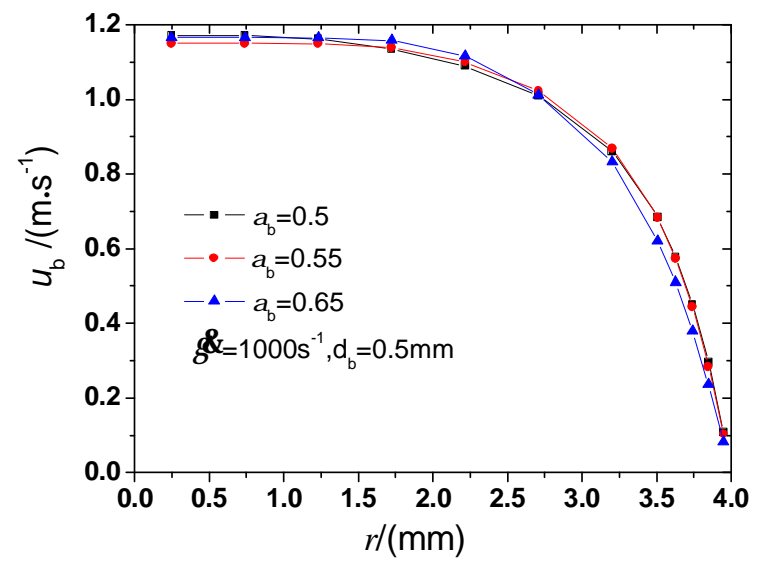

Fig.5. Velocity profile of granular phase

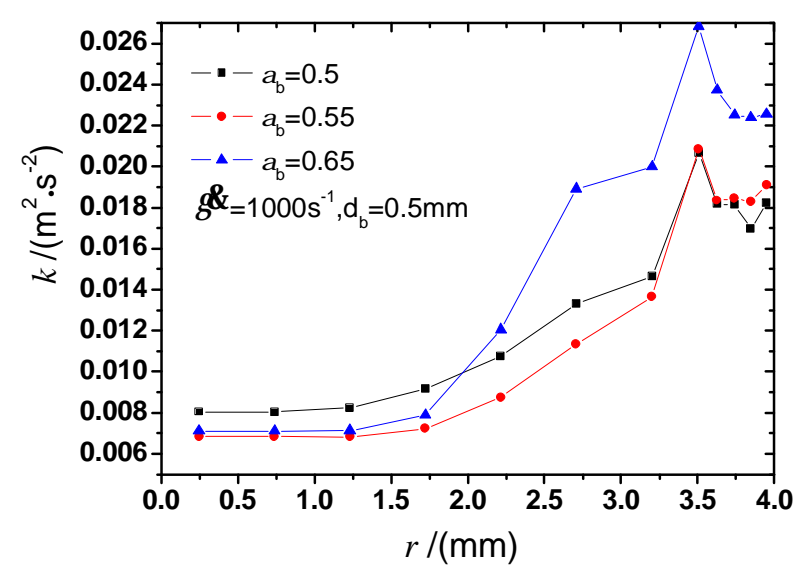

Fig.6. Turbulence pulsation kinetic-energy distribution of liquid phase

Turbulence pulsation kinetic-energy distribution

Figs. 6 and 7 show that the turbulence pulsation kinetic-energy of liquid and granular phase increases as foam quality increases. What's more, the turbulence pulsation kinetic-energy close to pipe wall is the highest. 


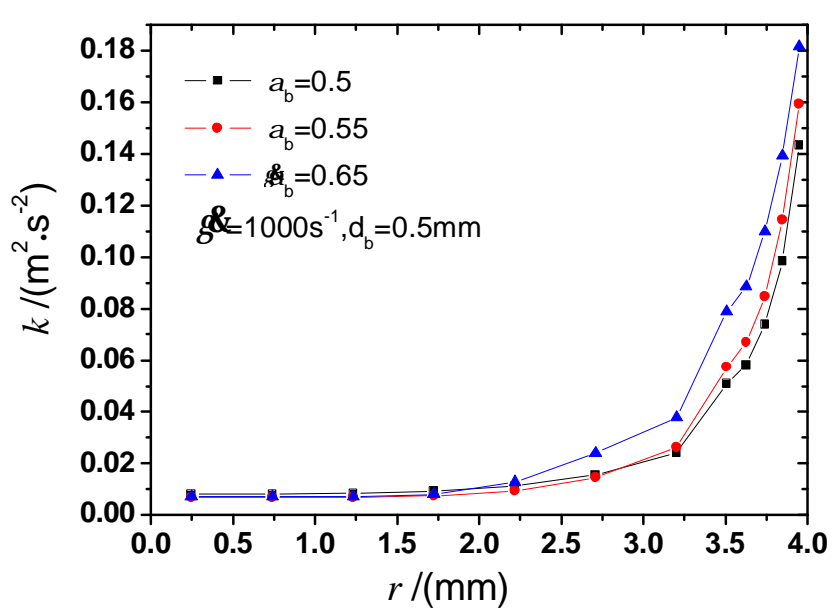

Fig.7. Turbulence pulsation kinetic-energy distribution of granular phase

\section{Conclusions}

In the model the bubble is handled as "granular". The simulating results show that the foam fluid is shear thinning, mainly because the bubble size decreases with the rise of shear rate. The viscosity and non-Newtonian fluid characteristic of foam fracturing fluid were influenced by the granular phase. Moreover, the increase in viscosity of foam fracturing fluid was mainly caused by the collision and friction between liquid phase and bubbles, and the friction takes a leading role in high foam quality. Furthermore, there is little slip velocity between liquid phase and bubbles. The turbulent kinetic energy of each phase, which increases with the rise of volume fraction, has the largest value at the region close to pipe wall. However, the model is not applicable to foam fluid with foam quality higher than $65 \%$.

\section{References}

[1] Reidenbach V.G., Harris P.C., Lee Y.N., et al. Rheological study of foam fracturing fluids using nitrogen and carbon dioxide. SPE production Enginering 12026, 1986, p. 31-41

[2] Durian D.J. Foam mechanical at bubble scale, Phys. Rev. Lett. 75, 1995, p. 4780-4783.

[3] Yuan X.F., Edwards S.F. Flow behavior of two-dimensional random foam. J Non-Newt. Fluid Mech. 60, 1995, p.335- 348.

[4] Simon C., Denis W. James A.G.The rheology of two-dimensional foams. Rheol. Acta. 43 , 2004, p. $442-448$.

[5] Kern N., Weaire D., Martin A., et al. Two-dimensional viscous froth model for foam dynamics. Phys. Rev. E. 70, 1-13

[6] Sun Q. and Hutzler S. Lattice gas simulations of two- dimensional liquid foams. Rheol. Acta. 43, 2004, p. 567-574

[7] Pozrikidis C. Numerical investigation of the effect of surfactants on the stability and rheology of emulsions and foam. J. Engng. Math. 41, 2001, p. 237- 258. 